\title{
Local-field effects in current transport through molecular electronic devices: Current density profiles and local non-equilibrium electron distributions
}

\author{
Yongqiang Xue * and Mark A. Ratner \\ Department of Chemistry and Materials Research Center, \\ Northwestern University, Evanston, Illinois 60208, USA
}

(Dated: May 11, 2018)

\begin{abstract}
We analyze non-equilibrium current transport in molecular electronic devices, using as an example devices formed by two terphenyl dithiol molecules attached to gold electrodes. Using a first-principles based self-consistent matrix Green's function method, we show that the spatially resolved current density profiles and local electrochemical potential drops provide valuable information regarding the local field effect on molecular transport, which depend on the internal structure of the molecules and cannot be obtained from measuring the current- and conductance-voltage characteristics alone.
\end{abstract}

PACS numbers: 85.65.+h,73.63.-b,73.40.-c

Introduction.- Detailed understanding of electron transport through single molecules contacted by metallic electrodes is crucial for the development of the emerging technology of molecular electronics. $\frac{1}{1}$ Although much attention has been devoted to the measured molecular conductance and its dependence on metal-molecule interactions,$\frac{1}{\underline{1}}$ less is known about the non-equilibrium aspects of the phase-coherent molecular transport, which provide information not obtainable from the conductance measurement 2.3 .4

Several important effects are of interest for nonequilibrium transport: (1) A finite electric field associated with the applied bias voltage will induce charge pileup (screening) within the molecular junction. The local transport field experienced by the tunneling electron is the self-consistent screened field; (2) Since the total current is conserved throughout the molecular junction for dc-transport, the spatial distribution of the current density can be highly non-uniform since the molecules are intrinsically inhomogeneous; (3) As current flows, the electrons at the source and drain contacts have different electrochemical potentials. It is not clear if an effective local electrochemical potential (LEP) can be defined everywhere within the molecular junction to characterize the electron distribution in the non-linear transport regime.

The screened nature of the local transport field has been analyzed in previous works,$\stackrel{3.4}{,}$ following early ideas of Landauer and Buttiker in mesoscopic transport systems. ${ }^{5}$ In particular, resistivity dipoles and strong local fields have been found in the vicinity of potential barriers both at the interface and at certain points inside the molecules. These in general modify both the energy and wavefunction of the frontier molecular states and lead to different electrostatic potential drops depending on the molecules and the device structures. ${ }^{3}$ The non-equilibrium electron distribution within the molecular junction has also been analyzed by projecting the nonequilibrium density-matrix into individual molecular orbitals ${ }^{3}$ Here, we extend previous work by presenting real-space analysis of current density profiles and local non-equilibrium electron distributions throughout the molecular junctions. Such analysis has important implications in resolving the following issues: (1) The identification of current path gives a clear picture of the conducting and non-conducting part of the molecular junction. In particular, large current density may develop at certain parts of the molecule, which forms a bottleneck to transport and might possibly be imaged using scanned probe microscopes $;{ }^{6}$ (2) The local electrochemical potential (LEP) characterizes the energy-distribution of the electrons everywhere within the junction, and is relevant in multi-probe transport measurement using a weakly coupled phase-sensitive voltage probe,,$\frac{7}{\underline{H}}$ e.g., in scanning tunneling potentiometry (STP). fined here is determined by the local density of states (LDOS) of the molecular junction in the presence of current transport, which may possibly be probed using scanning tunneling spectroscopy (STS) 9,10

Non-Equilibrium Green's Function (NEGF) approach to scattering theory of molecular transport.-We analyze the non-equilibrium effects within the scattering theory of mesoscopic transport, 11 where the relevant physical quantities are obtained from microscopic calculations using a self-consistent matrix Green's function theory ${ }^{3.12}$ which combines the NEGF theory ${ }^{13}$ with an effective single-particle description of molecular junction electronic structure using density functional theory ${ }^{14} \mathrm{We}$ use atomic unit throughout the paper unless otherwise noted.

The central quantity within the NEGF theory is the correlation Green's function $G^{<}\left(\vec{r}, \vec{r}^{\prime} ; E\right)=$ $\sum_{i j} G_{i j}^{<}(E) \phi_{i}(\vec{r}) \phi_{j}\left(\vec{r}^{\prime}\right)$, where we have expanded $G^{<}$ in terms of local atomic basis sets $\phi_{i}$ (which are real functions) 12 The current density is obtained from $G^{<}\left(\vec{r}, \vec{r}^{\prime} ; E\right)$ as 13

$\vec{j}(\vec{r})=1 / 2 \lim _{\vec{r}^{\prime} \rightarrow \vec{r}}\left(\nabla^{\prime}-\nabla\right) \int d E G^{<}\left(\vec{r}, \vec{r}^{\prime} ; E\right)=1 / 2 \sum_{i, j}\left(\int d E G_{i, j}^{<}(E)\right) d$

where we used the notation $d S_{i j}(\vec{r})=\phi_{i}(\vec{r}) \nabla \phi_{j}(\vec{r})-$ $\phi_{j}(\vec{r}) \nabla \phi_{i}(\vec{r})$. The terminal current is obtained by integrating the current density over a surface separating the molecule from the con- 

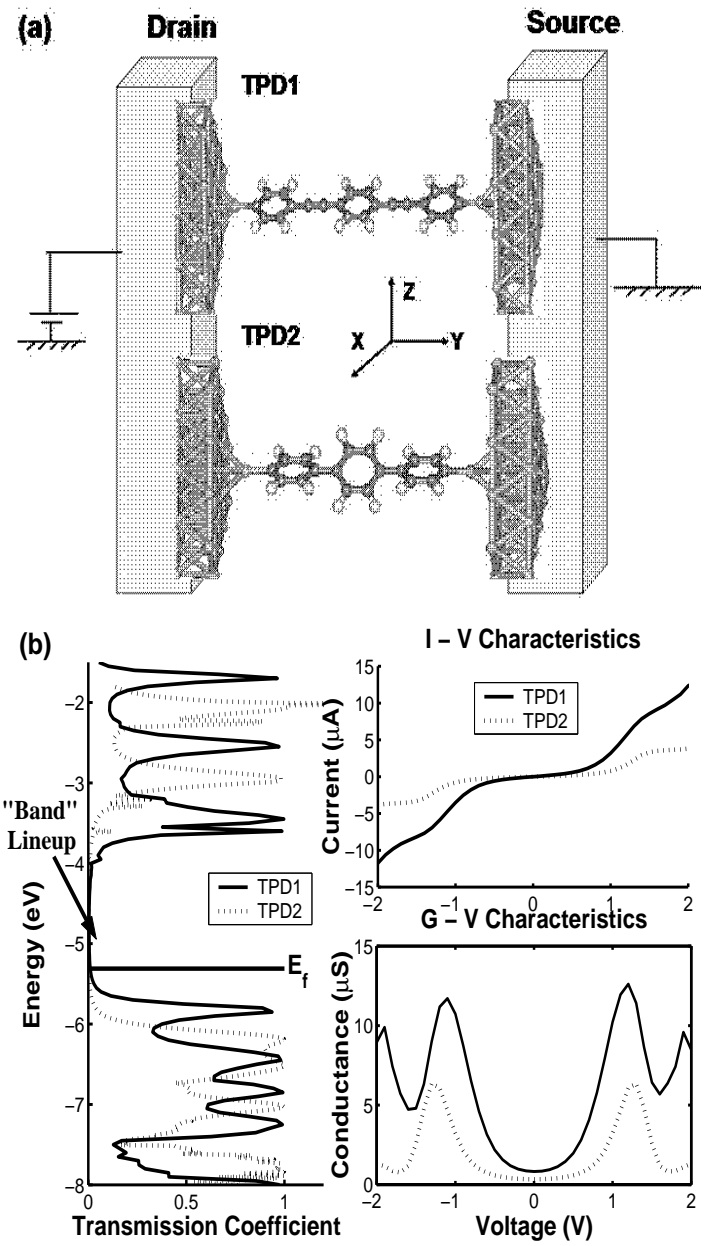

FIG. 1: (Color online) (a) Schematic illustration of the metal-molecule-metal junction for the two terphenyl dithiolate (TPD) molecules. Six gold atoms on each metal surface (12 overall) are included into the "extended molecule" where the self-consistent calculation is performed. The effect of the rest of the electrodes (with the 6 atoms on each side removed) are modeled as self-energy operators. Also shown is the bias polarity and coordinate system of the molecular junction; (b) The left figure shows the equilibrium electron transmission coefficent. The right figures show the self-consistent current-voltage (I-V) and differential conductance-voltage (G$\mathrm{V})$ characteristics of the two molecular junctions.

tact, which leads to the standard formula11.12.13 $I_{L(R)}=\int d E \operatorname{Tr}\left\{\Gamma_{L(R)}\left[f\left(E-\mu_{L(R)}\right) A(E)+i G^{<}(E)\right]\right\}=$ $\int d E \int d \vec{r}\left\{\Gamma_{L(R)}(\vec{r}, \vec{r}) \sum_{i j}\left[f\left(E-\mu_{L(R)}\right) A(\vec{r}, \vec{r} ; E)+\right.\right.$ $\left.\left.i G^{<}(\vec{r}, \vec{r} ; E)\right]\right\}$, where $A(\vec{r}, \vec{r} ; E)=\sum_{i j} A_{i j}(E) \phi_{i}(\vec{r}) \phi_{j}(\vec{r})$. The spectral function $A$ and the operators $\Gamma_{L(R)}$ describing the contacts are defined in the standard manner 11.12.13 Note that in the matrix notation, we have $A=G^{R}\left[\Gamma_{L}+\Gamma_{R}\right] G^{A}=A_{L}+A_{R}$ and $-i G^{<}=G^{R}\left[\Gamma_{L} f_{L}+\Gamma_{R} f_{R}\right] G^{A}=A_{L} f_{L}+A_{R} f_{R}$, where $A_{L(R)}=G^{R} \Gamma_{L(R)} G^{A}$. Defining a local transmission function for electron injection from the left (right) electrode $T_{L(R)}(\vec{r} ; E)=$ (a)

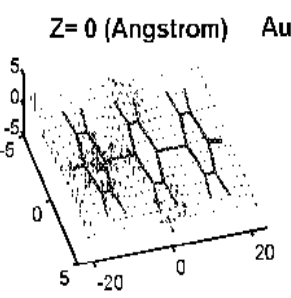

Au-TPD1-Au $Z=1.5$ (Angstrom)
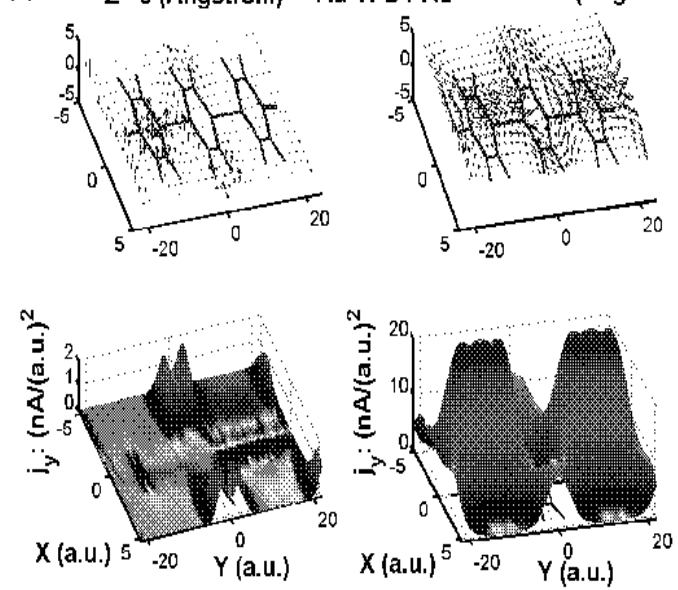

(b)
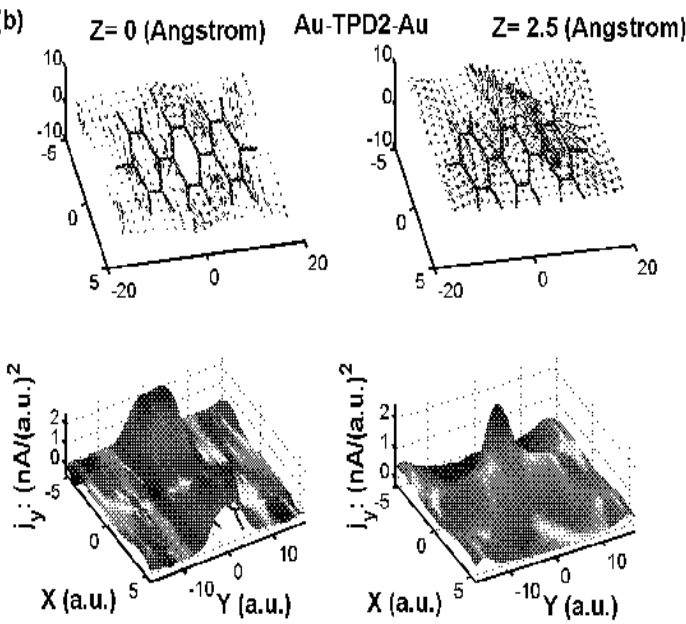

FIG. 2: (Color online) Cross sectional view of current density profiles at gold-TPD1-gold (a) and gold-TPD2-gold (b) junctions. Also shown is the position of the molecules. For each junction, the upper figures show the direction of current density, the lower figures show the magnitude of current density component along the transport direction ( $Y$ axis here).

$\Gamma_{L(R)}(\vec{r}, \vec{r} ; E) A(\vec{r}, \vec{r} ; E)$, the total current can be expressed in terms of $T_{L(R)}(\vec{r} ; E)$ and an effective local electron-distribution function $f_{\text {eff }}(\vec{r} ; E)$ as: $I_{L(R)}=\frac{e}{h} \int d E \int d \vec{r} T_{L(R)}(\vec{r} ; E)\left[f_{L(R)}(E)-f_{e f f}(\vec{r} ; E)\right]$, where $A(\vec{r}, \vec{r} ; E) f_{\text {eff }}(\vec{r} ; E)=-i G^{<}(\vec{r}, \vec{r} ; E)=$ $A_{L}(\vec{r}, \vec{r} ; E) f_{L}(E)+A_{R}(\vec{r}, \vec{r} ; E) f_{R}(E)$.

Replacing the spectral functions by the corresponding density of states $A(\vec{r}, \vec{r} ; E)=2 \pi \rho(\vec{r} ; E), A_{L(R)}(\vec{r}, \vec{r} ; E)=$ $2 \pi \rho_{L(R)}(\vec{r} ; E)$, we arrive at the following definition of an effective local non-equilibrium electron distribution function: $f_{\text {eff }}(\vec{r} ; E)=\frac{\rho_{L}(\vec{r} ; E)}{\rho(\vec{r} ; E)} f_{L}(E)+\frac{\rho_{R}(\vec{r} ; E)}{\rho(\vec{r} ; E)} f_{R}(E)$. At low temperature and within the linear-response regime, we can replace the Fermi distributions by step-like functions and define the local electrochemical potential (LEP) as $\mu_{e f f}(\vec{r})=\frac{\rho_{L}\left(\vec{r} ; E_{f}\right)}{\rho\left(\vec{r} ; E_{f}\right)} \mu_{L}+\frac{\rho_{R}\left(\vec{r} ; E_{f}\right)}{\rho\left(\vec{r} ; E_{f}\right)} \mu_{R}$ where $E_{F}$ is the equilibrium Fermi-level. At finite temperature in the nonlinear transport regime, the above definition of LEP re- 

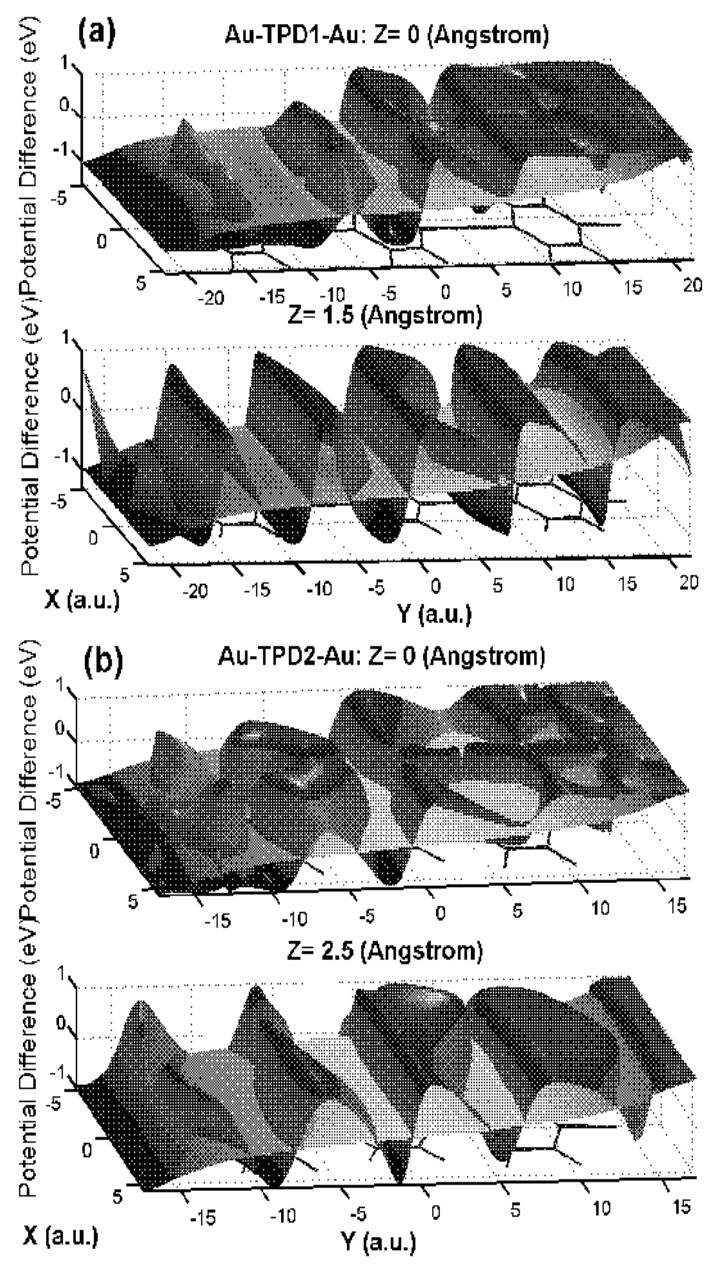

FIG. 3: (Color online) Cross sectional view of local electrostatic potential and electrochemical potential drops at the gold-TPD1-gold (a) and gold-TPD2-gold (b) junctions. Also shown are the positions of the molecules. The electrostatic potential varies rather smoothly from $-V / 2$ to $V / 2$ across the junction, but the electrochemical potential oscillates strongly between $-V / 2$ and $V / 2$.

mains useful for understanding transport physics if the coefficients $\frac{\rho_{L(R)}(\vec{r} ; E)}{\rho(\vec{r} ; E)}$ vary slowly with energy between $\mu_{L}$ and $\mu_{R}$. Since this is true for molecules chemisorbed onto the metal surfaces, we get the following LEP defined everywhere within the molecular junction:

$\mu_{e f f}(\vec{r})=\frac{\sum_{i j} \rho_{L ; i j}\left(E_{f}\right) \phi_{i}(\vec{r}) \phi_{j}(\vec{r})}{\sum_{i j} \rho_{i j}\left(E_{f}\right) \phi_{i}(\vec{r}) \phi_{j}(\vec{r})} \mu_{L}+\frac{\sum_{i j} \rho_{R ; i j}\left(E_{f}\right) \phi_{i}(\vec{r}) \phi_{j}}{\sum_{i j} \rho_{i j}\left(E_{f}\right) \phi_{i}(\vec{r}) \phi_{j}}$

Note that an equivalent definition of the local electrochemical potential has been given by Gramespacher and Büttiker ${ }^{15}$ for a mesoscopic conductor within the scattering matrix theory. Here the contact-resolved local density-of-states $\rho_{L(R)}=G^{R} \Gamma_{L(R)} G^{A} / 2 \pi$ is proportional to the injectivity of the left (right) electrode given by Gramespacher and Büttiker. The LEP $\mu_{e f f}(\vec{r})$ as defined here in a current-carrying conductor may possibly be measured by an ideal phase-sensitive non-invasisve voltage probe (Büttiker probe) introduced at point $\vec{r} \cdot 7.15$ In scanning tunelling potentiometry, this is identified with the bias voltage between the STP tip and the sample under the condition of zero tunneling current 7.8

Results and their interpretation.-Since local-field effects are most clearly seen for longer molecules with internal barriers, $\stackrel{3}{=}$ we apply our theory to the devices formed by two extended $\pi$ systems-a three-ring oligomer of phenylene ethynylene with dithiol substituents (called TPD1) and a terphenyl dithiol molecule (called TPD2)in contact with gold electrodes through the end sulfur atoms. The device structure is illustrated schematically in Fig. 1(a). The benzene rings are co-planar when connected by a triple-bonded C-C bridge (TPD1), which leads to optimal orbital overlap. In the absence of the C-C bridge (TPD2), a torsion angle of $36^{\circ}$ is induced between neighbor benzene rings, which leads to weaker orbital overlap and an effective potential barrier for electron motion inside the molecule 3 The calculation is performed using a modified version of Gaussian98 program ${ }^{16}$ with the Becke-Perdew-Wang parameterization of density-functional theory ${ }^{14}$ and appropriate pseudopotentials with corresponding optimized Gaussian basis sets. The details of the self-consistent calculation have been discussed extensively elsewhere ${ }^{3.17}$

The equilibrium (zero bias) electron transmission characteristics of the molecular junctions are shown in Fig. 11(b), from which we find that for both molecules, the highest-oocupied-molecular-orbital (HOMO) lines up closer to the metal Fermi-level $E_{F}$ than the lowestunoccupied-molecular-orbital (LUMO), similar to previous finding on phenyl and biphenyl dithiolate molecules. ${ }^{3}$ The lineup scheme is more favorable for the planar TPD1 molecule. The room-temperature conductance of the molecular junction is $0.82(\mu S)$ and $0.34(\mu S)$ for TPD1 and TPD2 junctions respectively. The self-consistent current-voltage (I-V) and differential conductance-voltage $(\mathrm{G}-\mathrm{V})$ characteristics in the bias range of $-2(V)$ to $2(V)$ are shown in Fig. 1(b). Note that despite the $\geq 0.3(\mathrm{eV})$ difference in the HOMO level position with respect to $E_{F}$ at equilibrium, there is only a $0.1(V)$ difference in the bias voltage where the junction reaches the first conductance peak $(1.2(V)$ and $1.3(V)$ for the TPD1 and TPD2 junction respectively). This is due to the stronger bias-induced modification of molecular states (static Stark effect) in the TPD2 molecule, $\phi_{j i \vec{i} \vec{n})}$ ce the internal barrier across the neighbor benzene $(\overrightarrow{\text { vin}}) \mu_{\mathrm{S}} R_{\text {leads }}$ to stronger local-field variations inside the molecule core. This pushes the HOMO up relative to the equilibrium Fermi-level and correspondingly reduces the voltage needed to move the metal Fermi-levels past the HOMO level.

The overall I-V and G-V characteristics of the two molecules show similar behavior, but the spatiallyresolved current density distributions are quite different due to the different molecular structures, which are shown in Fig. 2 for the two molecules at bias voltage 
of $2(V)$. Note that the direction of current is reverse to that of the net electron density flux due to the negative electron charge. For the planar TPD1 molecule, we show both the vectorial plot (direction) of current density and the magnitude of the y-component (perpendicular to the electrode surface) of current density $j_{y}$ in the $x y$-plane (defined by the benzene rings) and in the plane located $1.5(\AA)$ above. For the nonplanar TPD2 molecule, this is shown in the $x y$-plane (defined by the left-most and right-most benzene rings) and in the plane located 2.5 $(\AA)$ above (the carbon and hydrogen atoms in the central benzene ring are located at $Z= \pm 0.7(\stackrel{\circ}{A})$ and $\pm 1.3(\stackrel{\circ}{A})$ respectively).

For the planar TPD1 molecule, the small y-component of the current density in the $x y$ plane peaks around the peripheral hydrogen atoms of the central benzene ring. $j_{y}$ increases rapidly (and symmetrically) moving above/under the benzene plane, peaks near $0.8(\AA)$ from it and then decreases slowly with further increase of $|Z|$. Note that the peak location of $j_{y}$ corresponds to the peak location of the density of the $\pi$ electrons in the molecule. The direction of the dominant ( $\pi$-electron) current flow is such that electrons are injected from the right electrode mostly along the sulfur-surface bond, and then propagate through the middle of the right-most benzene ring (centered around $Y$-axis), being diverted to the perimeters of the central benzene, and converge again into the middle of the left-most benzene ring. For the nonplanar TPD2 molecule, there is a large $j_{y}$ in the plane $Z=0$, which resides mostly around the central benzene ring. Moving away from $Z=0, j_{y}$ increases gradually in both the left and right benzene rings, and reaches its peak at about $0.7(\stackrel{\circ}{A})$, corresponding to the position of the carbon atoms in the central benzene (the peak value is only about three times that in the $X Y$ plane) and then decreases slowly. For positive (negative) $Z, j_{y}$ is larger on the negative (positive) $X$ half of the junction, determined by the location of carbon and hydrogen atoms in the central benzene ring. The direction of the dominant current flow is such that electrons propagate rather uniformly through the right-most benzene ring, being diverted towards the negative (positive) $X$ half of the molecule at positive (negative) $Z$ and propagate again rather uniformly through the left benzene ring.

The local electrostatic potential and electrochemical (LEP) potential drops in the molecular junction are shown in Fig. 3 for bias voltage of $2(V)$. Unlike the electrostatic potential drop which varies rather smoothly from $-V / 2$ to $V / 2$ across the junction, the local electrochemical potential shows oscillatory behavior between $-V / 2$ and $V / 2$ throughout the molecular junction. This is due to the phase-sensitive nature of the voltage probe: an electron wave incident from either electrode gives two contributions to the electron flux in the voltage probe at point $\vec{r}$, i.e., the direct transmission and the transmission after multiple scattering within the molecular junction. The oscillatory behavior in LEP therefore demonstrates the presence of a potential barrier for electron injection into the molecular junction. The more opaque (smaller transmission coefficient) the barrier is, the larger the magnitude of the oscillation will be ${ }^{7}$ The oscillation of the LEP is also sensitive to the shape of the potential barriers, which shows up clearly in the two planes of the molecular junctions (Fig. 3). The LEP in the TPD2 junction oscillates more strongly than the TPD1 junction due to the presence of barriers both at the metal-molecule interface and inside the molecule induced by the weaker orbital overlap across neighbor benzene rings. In addition, there is also oscillation in the direction (along $X$ axis) perpendicular to the transport direction due to the three-dimensional structure of the molecular junction.

Discussion and Conclusion. Although in principle both the local electrochemical potential (LEP) and the current density profile in single-molecule devices may possibly be measured using scanning nanoprobe techniques, in practice this can be extremely difficult due to the spatial resolution involved and the requirement that the local probe should be minimally invasive. For example, the LEP varies on the scale of the Fermi wavelength $\left(\lambda_{F}\right)$ of electrons injected from the electrodes, which is $\sim$ 1(A) for metallic electrodes, unlike the two-dimensionalelectron-gas fabricated from semiconductor heterostructures where $\lambda_{F}$ can be of $100(\mathrm{~nm})$ and longer 18 Further work is therefore needed for a quantitative evaluation taking into account realistic experimental conditions in such multi-probe transport measurements, in particular the probe geometry effect and local probe-induced perturbation of the junction electronic processes. The present work hightlights the importance of local atomicscale analysis in revealing subtle effects in single-molecule electronic devices.

This work was supported by the DARPA Moletronics program, the DoD-DURINT program and the NSF Nanotechnology Initiative.
* Author to whom correspondence should be addressed. Email: ayxue@chem.northwestern.edu

1 M.A. Reed, Proc. IEEE 97, 652 (1999); C. Joachim, J.K. Gimzewski, and A. Aviram, Nature 408, 541 (2000); A. Nitzan and M.A. Ratner, Science 300, 1384 (2003); A. Salomon et al. Adv. Mater. 15, 1881 (2003).

2 M. Büttiker, J. Phys.: Condens. Matter 5, 9361 (1993).

3 Y. Xue and M.A. Ratner, Phys. Rev. B 68, 115406 (2003);
68, 115407 (2003); 69, 85403 (2004).

4 See also e.g. M. Di Ventra, S.T. Pantelides, and N.D. Lang, Phys. Rev. Lett. 84, 979 (2000); N.D. Lang, Phys. Rev. B 64, 235121 (2001); J. Taylor, H. Guo, and J. Wang, ibid. 63, 245407 (2001); P.S. Damle, A.W. Ghosh and S. Datta, ibid. 64, 201403 (2001); M. Brandbyge, J.-L. Mozos, P. Ordejón, J. Taylor, and K. Stokbro, ibid. 65, 165401 (2002); J. Heurich, J.C. Cuevas, W. Wenzel, and G. Schön, Phys. 
Rev. Lett. 88, 256803 (2002).

${ }^{5}$ R. Landauer, IBM J. Res. Develop. 32, 306 (1988); M. Büttiker, ibid. 32, 317 (1988).

6 M.A. Topinka, R.M. Westervelt, and E.J. Heller, Phys. Today 56(12), 47 (2003).

7 M. Büttiker, Phys. Rev. B 40, 3409 (1989); C.S. Chu and R.S. Sorbello, ibid. 42, 4928 (1990); M.J. McLennan, Y. Lee, and S. Datta, ibid. 43, 13846 (1991).

8 P. Muralt and D.W. Pohl, Appl. Phys. Lett. 48, 514 (1986); J.R. Kirtley, S. Washburn, and M.J. Brady, Phys. Rev. Lett. 60, 1546 (1988).

9 G.V. Nazin, X.H. Qiu, and W. Ho, Science 302, 77 (2003); W. Ho, J. Chem. Phys. 117, 11033 (2002).

${ }^{10} \mathrm{Ph}$. Avouris, I.-W. Lyo, and Y. Hasegawa, IBM J. Res. Develop. 39, 603 (1995).

11 S. Datta, Electron Transport in Mesoscopic Systems (Cambridge University Press, Cambridge, 1995).

12 Y. Xue, S. Datta, and M.A. Ratner, Chem. Phys. 281, 151 (2002).

13 Y. Meir and N.S. Wingreen, Phys. Rev. Lett. 68, 2512 (1992); H. Haug and A-P. Jauho, Quantum Kinetics in Transport and Optics of Semiconductors (Springer-Verlag, Berlin, 1996).

14 A.D. Becke, Phys. Rev. A. 38, 3098 (1988); M. Ernzerhof, J.P. Perdew and K. Burke, in Density Functional Theory $I$, edited by R. F. Nalewajski (Springer, Berlin, 1996).

15 T. Gramespacher and M. Büttiker, Phys. Rev. B 56, 13026 (1997).
16 GAUSSIAN 98, Revision A.7, M. J. Frisch et al, Gaussian, Inc. , Pittsburgh, PA, 1998.

17 To summarize, we assume here that the geometry of the molecules in the junction are the same as the isolated molecule, which are obtained by optimization at the UBPW91/6-31G* level. The adsorption geometry is such that the end sulfur atom sits in front of the center of the triangular gold-pad on the $\langle 111\rangle$ surface (parallel to the $X Z$-plane) with a end atom-surface distance of $1.9(\AA)$. For the atoms in the molecule, we use the pseudopotential and the corresponding polarized split valence basis sets of W.J. Stevens, H. Basch and M. Krauss, J. Chem. Phys. 81, 6026 (1984); For surface gold atoms, we use the pseudopotential and the valence basis sets (with the most diffuse component removed) of P.J. Hay and W.R. Wadt, J. Chem. Phys. 82, 270 (1985).

18 Scanning probe measuement of local voltage drop across a metal-carbon nanotube-metal junction has been reported recently by Y. Yaish, J.-Y. Park, S. Rosenblatt, V. Sazonova, M. Brink, and P.L. McEuen, Phys. Rev. Lett. 92, 46401 (2004). However, due to the large spatial resolution (tens of $\mathrm{nm}$ ) there, the measured electrochemical potential drop is the result of spatially-averaging over many oscillation periods of the local electrochemical potential, which essentially follows the local electrostatic potential drop. 